
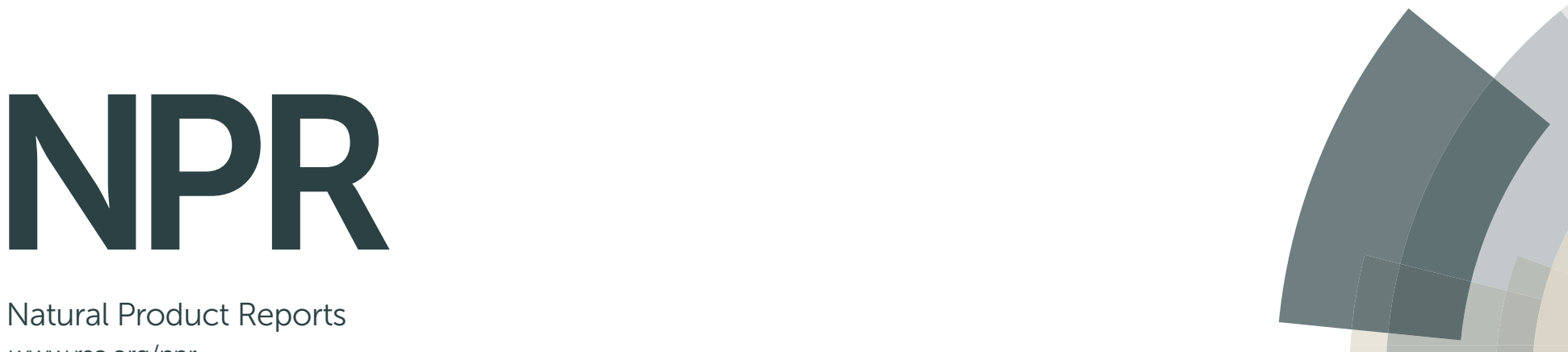

Natural Product Reports www.rsc.org/npr

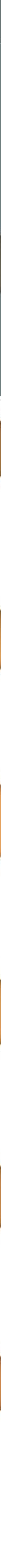

ISSN 0265-0568 


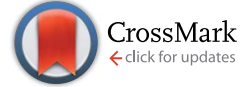

Cite this: Nat. Prod. Rep., 2015, 32, 765

\section{Involvement of secondary metabolites in the pathogenesis of the American foulbrood of honey bees caused by Paenibacillus larvae}

\author{
Sebastian Müller, ${ }^{a}$ Eva Garcia-Gonzalez, ${ }^{b}$ Elke Genersch *b \\ and Roderich D. Süssmuth*a \\ Covering: 2011 to end of 2014
}

The Gram-positive, spore-forming bacterium Paenibacillus larvae ( $P$. larvae) is the causative agent of the epizootic American Foulbrood (AFB), a fatal brood disease of the western honey bee (Apis mellifera). AFB is one of the most destructive honey bee diseases since it is not only lethal for infected larvae but also for the diseased colonies. Due to the high impact of honey bees on ecology and economy this epizootic is a severe and pressing problem.

Received 5th December 2014 Knowledge about virulence mechanisms and the underlying molecular mechanisms remain largely elusive. Recent genome sequencing of $P$. larvae revealed its potential to produce unknown secondary metabolites, like nonribosomal peptides and peptide-polyketide hybrids. This article highlights recent findings on secondary metabolites synthesized by $P$. larvae and discusses their role in virulence and pathogenicity towards the bee larvae.
DOI: $10.1039 / c 4 n p 00158 c$

www.rsc.org/npr the etiological agent of the American Foulbrood (AFB). ${ }^{5}$ AFB is occurring worldwide and leads to massive losses of entire bee colonies every year. AFB is highly contagious and spreads very rapidly. In many countries, $\mathrm{AFB}$ is a notifiable disease and infected bee hives have to be burned to contain the disease. An effective treatment of AFB does still not exist, since the underlying molecular characteristics of the $P$. larvae infection remain largely elusive. Therefore, it is difficult to develop efficient approaches to fight this severe epizootic.

Based on repetitive element PCR (repPCR) using enterobacterial repetitive intergenic consensus sequence (ERIC) primers, ${ }^{6}$ the species $P$. larvae has been classified into four different genotypes (ERIC I-IV) which could recently be confirmed by multi locus sequence typing (MLST) of hundreds of $P$. larvae isolates from all over the world. ${ }^{5,7}$ These genotypes also differ phenotypically with respect to spore and colony morphology, metabolism, and most importantly virulence. ${ }^{\mathbf{5 , 8 9}}$ Therefore, the classification of the species into ERIC genotypes is of biological relevance. The genotypes ERIC I and ERIC II are regularly isolated from infected colonies worldwide, whereas ERIC III and ERIC IV are only represented by few historical isolates in type culture collections. ${ }^{10}$ Hence, only P. larvae ERIC I and ERIC II are of practical importance and will be covered in this review. Comparative exposure bioassays have demonstrated that the genotype ERIC II is more virulent on the larval level than ERIC I. It commonly kills bee larvae within 6-7 days, while ERIC I strains need up to 12 days to kill all infected larvae. ${ }^{\mathbf{1 1}}$ These differences on the individual larval level translate to differences in virulence on the colony level because the earlier larvae die the more efficiently they can be removed by nurse bees engaged in
${ }^{a}$ Institut für Chemie, Technische Universität Berlin, 10623, Berlin, Germany. E-mail: suessmuth@chem.tu-berlin.de

${ }^{b}$ Institute for Bee Research, Department of Molecular Microbiology and Bee Diseases, 16540, Hohen Neuendorf, Germany.E-mail: elke.genersch@hu-berlin.de 
brood hygiene as part of the social immune response of honey bees. ${ }^{12}$ The social immune response is better adapted to contain ERIC II infections than ERIC I infections because most ERIC IIinfected larvae die early and, hence, these are more efficiently removed from the colony than the slower dying ERIC I-infected larvae. This leads to the paradoxical situation that $P$. larvae ERIC II is less virulent on the colony level than ERIC I. ${ }^{8}$

From the bacterium $P$. larvae only the spores are infectious and only young larvae (up to the age of 36 hours after egg hatching) are susceptible to infection. ${ }^{\mathbf{4} 13}$ Hence adult honey

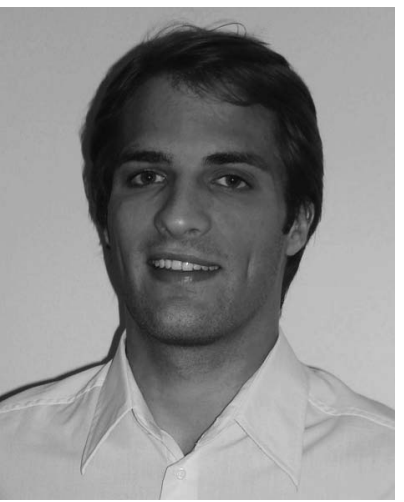

Sebastian Müller was born in 1983 and studied Biology at Goethe University in Frankfurt (Germany), where he received his diploma in Molecular Biology (2010). His PhD research at the Institut für Chemie of the Technische Universität Berlin with Prof. Roderich D. Süssmuth focuses on the secondary metabolites and biosynthetic pathways of the bee pathogenic bacterium Paenibacillus larvae.

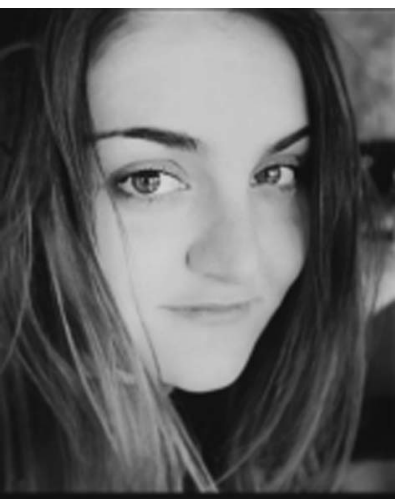

Eva García-González studied Biology at the Universitat de València (Spain), receiving her degree in 2006. Later, she worked at Sartorius-Stedim Biotech (Göttingen, Germany) where she completed a degree thesis in collaboration with the University of Göttingen, Germany. Afterwards, she joined the ZIBI Graduate School (Zentrum für Infektionsbiologie und Immunität) and started her PhD studies in Berlin dealing with host-pathogen interactions. As PhD student she worked at the Humboldt-Universität zu Berlin and the Institute for Bee Research (Hohen Neuendorf) as part of the Genersch group in the department for Molecular Biology and Pathology. She obtained her PhD degree in May 2013. Afterwards she worked as postdoc at the Bee research Institute and the Freie Universität Berlin. bees do not become infected by the uptake of $P$. larvae endospores. ${ }^{\mathbf{1 4 , 1 5}}$ Although only bee larvae are killed by $P$. larvae, the resulting lack of offspring ultimately leads to the collapse of the entire colony. In consequence weakened and collapsing colonies are likely robbed by strong colonies. Because the honey of $P$. larvae infected colonies is contaminated by $P$. larvae spores, bees robbing the food stores of such colonies efficiently transmit the disease to their own colonies.

The infection is initiated as soon as P. larvae spores are taken up by larvae via spore contaminated larval food and germinate

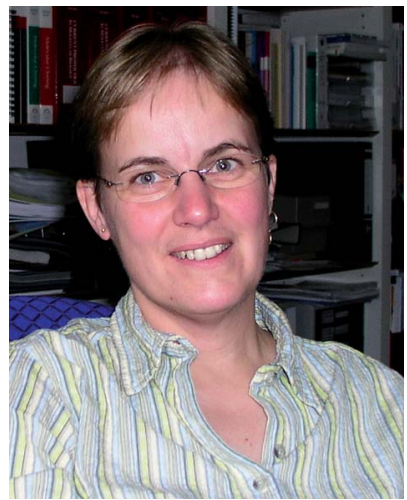

Elke Genersch is currently Vice Director of the Institute for Bee Research in Hohen Neuendorf, Germany, and Assistant Professor of Molecular Microbiology at the Freie Universität Berlin. She holds degrees in molecular biology from the University of Cologne (Diploma 1988), in biochemistry from the Ludwig-Maximilians-Universität Munich (doctoral degree 1993), and in molecular microbiology from the Freie Universität Berlin (habilitation 2006). After a short investment in industrial research she accepted group leader positions at the Max-Delbrück-Center for Molecular Medicine in Berlin-Buch (1994-1999) and at the Biomedical Center in Lund, Sweden, (1999-2001). In 2001, she took up the position as Vice Director of the Institute for Bee Research and started working on honey bee pathogens. Her research focuses on elucidating strategies and virulence factors used by viral, bacterial, and fungal pathogens to interact with the host, i.e. honey bee larvae, pupae, and adults.

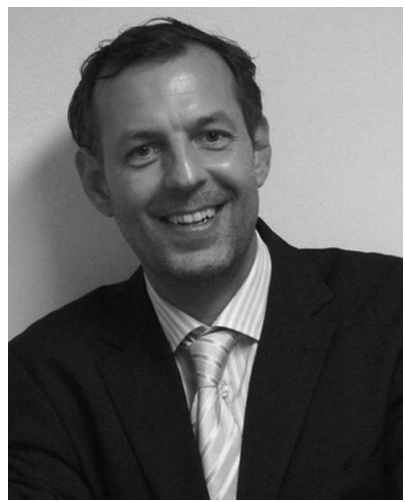

Roderich Süssmuth is the Rudolf Wiechert Professor of Biological Chemistry at the Technische Universität Berlin. He obtained his PhD degree in chemistry in 1999 from the Eberhard Karls Universität Tübingen with Günther Jung. In 2000, he was a Feodor-Lynen-Fellow of the Alexander von Humboldt-Foundation with Carlos Barbas III and Richard Lerner. Subsequently he became an Assistant Professor in Tübingen with an Emmy-Noether fellowship granted by the DFG, and then moved in 2004 to the TU Berlin. His research is focused on antibiotics, biosynthetic assembly lines, host-pathogen interactions, medicinal chemistry of peptides, enzymes and enzyme inhibitors. 


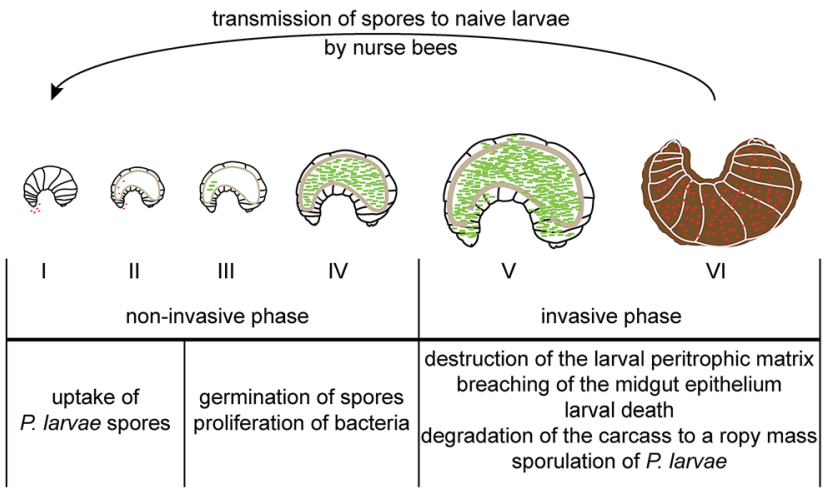

Fig. 1 Pathogenesis of $P$. larvae infections in honey bee larvae. The non-invasive phase of infections comprises the ingestion of $P$. larvae spores by naïve larvae through spore contaminated larval diet (I-II). The spores germinate in the larval midgut (III) where the vegetative bacteria proliferate massively until they occupy nearly the entire midgut lumen (IV). The invasive phase of infection is initiated by the total destruction of the midgut peritrophic matrix enabling the bacteria to attack and cross the epithelial barrier ( $V$ ). By then the infected larva is dead and $P$. larvae totally degrades the cadaver to a ropy mass before bacterial sporulation occurs. The ropy mass consisting of billions of spores dries down to the so called 'foulbrood scale'. Nurse bees trying to clean the brood cell become contaminated by these spores and transmit them to naïve larvae when feeding them. $P$. larvae secondary metabolites exhibiting antimicrobial activity might play a role during $P$. larvae proliferation in the midgut lumen (IV) by eliminating bacterial and fungal competitors and during the degradation of the larval remains to a ropy mass $(\mathrm{VI})$ by preventing saprophytes to assist in degradation.

in the larval midgut (Fig. 1). The vegetative bacteria then massively proliferate in the midgut lumen. During this noninvasive phase of infection, no damage to the epithelial cell layer can be observed ${ }^{16}$ although the protective peritrophic matrix (PM) might already be affected (Fig. $2 \mathrm{~A}$ and B). ${ }^{17}$ P. larvae is able to metabolize sugars ${ }^{18}$ and crystalline chitin,,${ }^{17}$ hence, the larval food and chitin containing larval structures like the PM can serve as nutrition during this phase of infection (Fig. 2B). ${ }^{17}$ The invasive phase of the infection starts when the PM is degraded $^{17,19}$ and the epithelial layer is attacked and penetrated by $P$. larvae (Fig. 2C and D). ${ }^{16}$ The bacteria invade the larval haemocoel (Fig. 1); a step that coincides with larval death. ${ }^{16}$ These characteristics of infection are shared by all P. larvae genotypes and the degradation of the larval PM has been shown to be a key step in AFB pathogenesis. ${ }^{1719}$ The chitin-degrading protein $P l$ CBP49 is responsible for PM degradation and is a unifying feature of pathogenesis reported to be expressed by both genotypes, ERIC I and ERIC II (Fig. 2B)..$^{19}$ In addition, genotype-specific virulence factors have been identified and it was proposed that $P$. larvae ERIC I and ERIC II follow different strategies when finally attacking and killing the infected larva (Fig. 2C and D)., ${ }^{9,20}$ The ERIC I-genome harbors several functional toxin genes which are disrupted and, hence, presumably non-functional in the ERIC II-genome. ${ }^{9}$ Two of the ERIC Ispecific toxins (Plx1, Plx2) have been characterized in more detail (Fig. 2C). ${ }^{21} \mathrm{Plx} 1$ and Plx2 are novel AB-toxins [for reviews on this class of toxins see Falnes and Sandvig ${ }^{22}$ and Simon

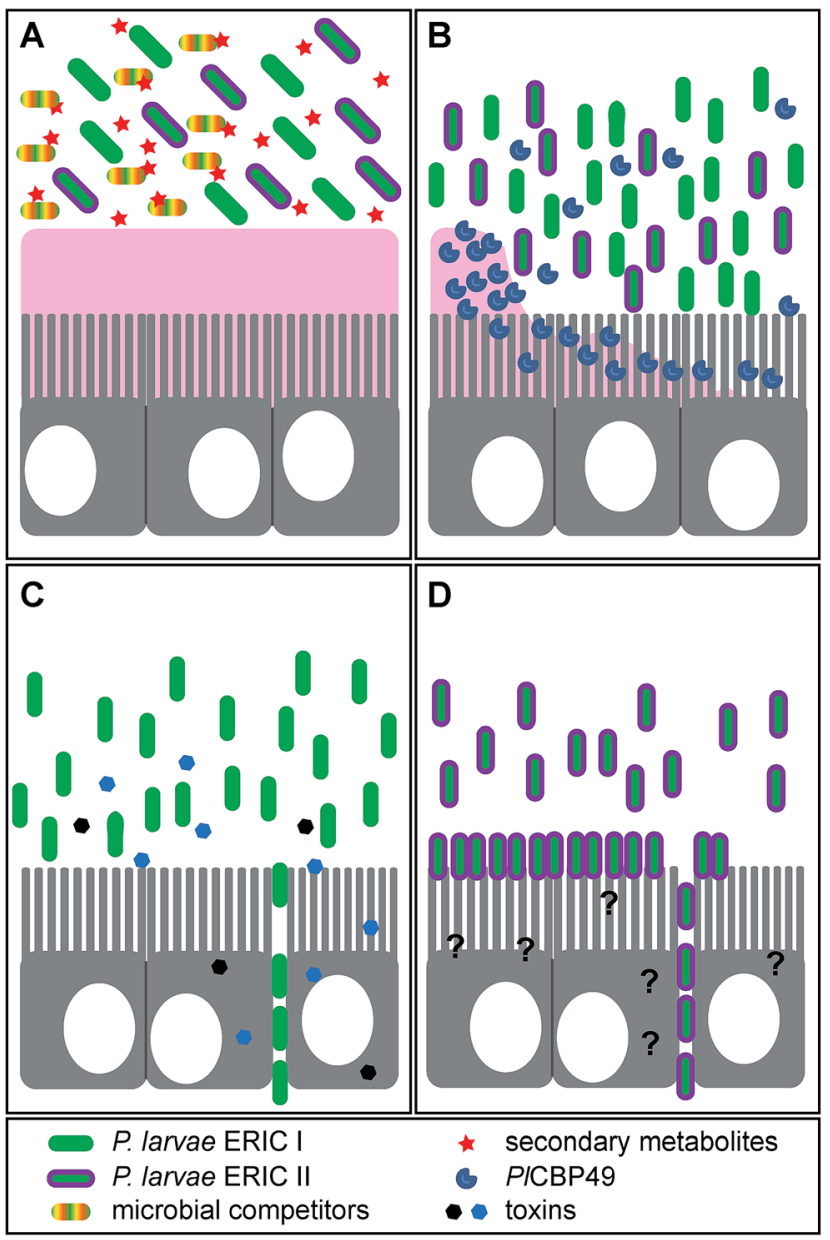

Fig. 2 Role of several recently identified virulence factors during pathogenesis of $P$. larvae infections. (A) $P$. larvae proliferating in the larval midgut will encounter microbial competitors soon the larval food is supplemented by honey and pollen both containing bacterial spores, bacteria, and fungi. Secondary metabolites like paenilamicin or the paenilarvins will enable $P$. larvae (i) to defend the niche "larval gut" and (ii) to outcompete saprophytes during degradation of the larval cadaver thus ensuring that a pure culture of $P$. larvae prevails in the end. (B) The larval midgut epithelium is protected against pathogen attack by a chitin-containing peritrophic matrix (pink structure in A and B). P. larvae expresses PICBP49, a novel, chitin-degrading enzyme. Total degradation of the peritrophic matrix by PICBP49 is the key step during pathogenesis of $P$. larvae infections and marks the transition from the non-invasive to the invasive life style of $P$. larvae. (C) two toxins, Plx1 and Plx2, are exclusively expressed by $P$. larvae ERIC I. For both, a role as virulence factor during pathogenesis has been experimentally demonstrated. These toxins most likely act on the epithelial cells, once the protective peritrophic matrix has been degraded. $P$. larvae then breaches the epithelial layer via the paracellular route and invades the hemocoel. By then the infected larva is dead and $P$. larvae starts to decompose the larval cadaver to a ropy mass. (D) The S-layer protein SplA is exclusively expressed by $P$. larvae ERIC II. SpIA mediates adhesion of $P$. larvae to the midgut epithelial cells, an important step during pathogenesis of $P$. larvae ERIC II infections. Most likely, degradation of the peritrophic matrix is a prerequisite for bacterial cell adhesion. Factors used by $P$. larvae ERIC II to accomplish breaching of the epithelium and invading the hemocoel are still elusive. Once the infected larva is dead, $P$. larvae starts to decompose the larval cadaver to a ropy mass. 
et $a l .{ }^{23}$ ] and their expression could be linked to virulence. ${ }^{21} \mathrm{Plx} 1$ is a single-chain $\mathrm{AB}$-toxin belonging to an enigmatic $\mathrm{AB}$-toxin family ${ }^{21}$ which also comprises MTX1 expressed by the entomopathogen Lysinibacillus sphaericus and the pierisin-like toxins expressed by some butterflies of the family Pieridae (Lepidoptera). ${ }^{24} \mathrm{Plx} 2$ is a binary AB-toxin and its A-moiety resembles C3like exoenzymes. ${ }^{21}$ The cellular targets of these novel AB-toxins still await identification. ${ }^{21}$ The ERIC II-genome harbors a functional S-layer protein gene ( $s p l \mathrm{~A})$ which is mutated and, hence, non-functional in the ERIC I-genome. ${ }^{25}$ Accordingly, expression of S-layer proteins could only be demonstrated in $P$. larvae ERIC II. $^{26}$ The $P$. larvae protein SplA was shown to mediate adhesion of $P$. larvae to primary bee midgut cells and expression of SplA could be linked to virulence of $P$. larvae ERIC II (Fig. 2D). ${ }^{25}$ The steps following $P$. larvae ERIC II adhesion to the midgut epithelium and those enabling the bacteria to penetrate the epithelial cell layer still remain elusive (Fig. 2D). ${ }^{25,26}$ These functionally characterized, genotypespecific virulence factors and genomic differences between $P$. larvae ERIC I and ERIC II clearly point to different virulence strategies pursued by ERIC I and ERIC II., ${ }^{9,20}$ However, irrespectively of the strategy, all P. larvae infections result in larval death and in the larval cadaver being converted in the brood cell to a ropy mass while non-infected, healthy larvae undergo metamorphosis and finally emerge as adult bees from the brood cell. Interestingly, this ropy mass is usually a pure culture of $P$. larvae,$^{27,28}$ suggesting that $P$. larvae has some mechanism to outcompete other bacteria and especially saprophytes that have been reported to be present in the larval gut. ${ }^{29}$ Therefore the recent identification of secondary metabolite gene clusters in the genome of $P$. larvae may contribute to facilitate interactions with potential competitors (Fig. 2A).

The species Paenibacillus larvae has originally been classified as Bacillus larvae. ${ }^{28}$ However, in 1991 the genus Bacillus was analyzed by comparative small-subunit rRNA (16S rRNA) sequence analysis and it was demonstrated that this genus comprised five phyletic lines, rRNA group 1-5 bacilli. $^{30} \mathrm{~A}$ subsequent extensive taxonomic revision revealed that the rRNA group 3 bacilli including Bacillus larvae were considerably distinct from the other Bacilli and, hence, they were reclassified as the new genus Paenibacillus. ${ }^{31}$ The genus Paenibacillus comprises several bacterial species formerly known as Bacilli like $P$. alvei and $P$. polymyxa. As the prefix Paeni reflects, Paenibacilli are 'almost' Bacilli and they share several features and properties with Bacilli. Bacilli are known to synthesize various secondary metabolites, such as lanthipeptides, lipopeptides, polyketides and other small molecules. ${ }^{32}$ Representatives of such compounds known for decades are the cyclic peptide antibiotics gramicidin $\mathrm{S}$ and tyrocidine, the lipopeptides surfactin and mycosubtilin and the siderophore bacillibactin. ${ }^{33-36}$ Like the genus Bacillus, Paenibacillus also seems to be an interesting source of bioactive natural products, although to date only few examples exist: $P$. polymyxa is known to produce polymyxin and fursaricidin ${ }^{37,38}$ and Paenibacillus elgii B69 produces the catechol-type siderophore paenibactin, which is closely related to the Bacillus siderophore bacillibactin. ${ }^{39}$ Furthermore, Paenibacillus sp. F6-B70 was described to produce the macrolide antibiotic paenimacrolidin, which is active against methicillin-resistant Staphylococcus aureus. ${ }^{\mathbf{4 0}}$ A cyclic lipopeptide, called battacin (octapeptin B5), was isolated from a Paenibacillus tianmuensis soil isolate and showed antibacterial activity against multidrug-resistant Gram-negative bacteria. ${ }^{\mathbf{4 1}}$

\section{Secondary metabolites from $P$. larvae}

It was not until the entire genome sequences of $P$. larvae ERIC I and ERIC II were available ${ }^{9}$ that the complex giant gene clusters coding for secondary metabolites in the genomes of $P$. larvae ERIC I and ERIC II could be identified and correctly assembled (Fig. 3). ${ }^{9}$ Sequencing and annotation of the genomes of $P$. larvae strain DSM25430 (genotype ERIC II; 4056006 bp) and P. larvae strain DSM25719 (genotype ERIC I; 4579589 bp) facilitated the bioinformatics analysis of the genes, particularly of those being involved in pathogenesis and secondary metabolite production
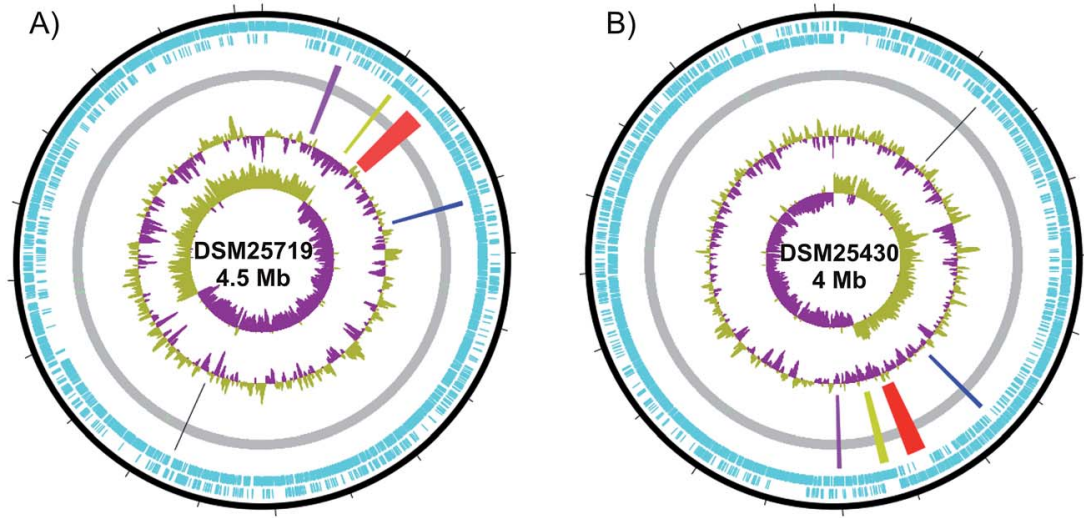

Fig. 3 Graphical circular maps of the chromosome of Paenibacillus larvae. (A) ERIC I DSM25719 and (B) ERIC II DSM25430. Open reading frames on the forward and reverse strand are shown in blue. The inner circles show first GC\% content and GC skew $[(G-C) /(G+C)]$ purple and olive indicating higher and lower values than average, respectively. NRPS and PKS gene clusters are highlighted from the CDS grey bar. In ERIC II (B) Black: monomodular NRPS, blue: sevadicin gene cluster, red: paenilamicin gene cluster, yellow: paenilarvin gene cluster and purple: bacillibactin gene cluster. In ERIC I (A) the correspondent gene clusters are shown in the same colors. Circular maps were drawn using dnaplotter. ${ }^{81}$ 
A)

ERIC I and II:

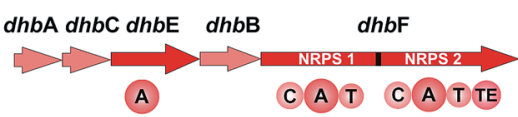

B)

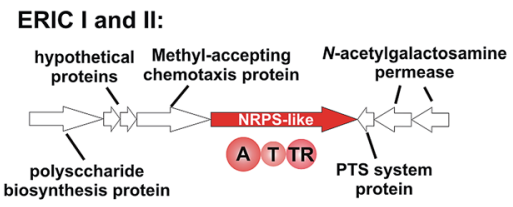

C)

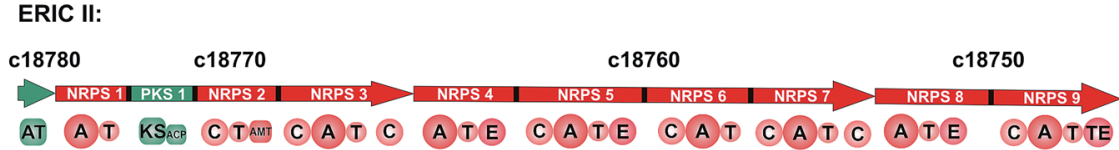

ERIC I:

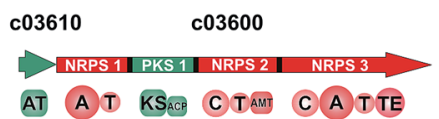

D)

ERIC II:

ERIC I:
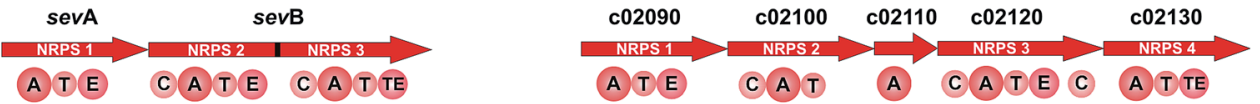

E)

ERIC I and II:
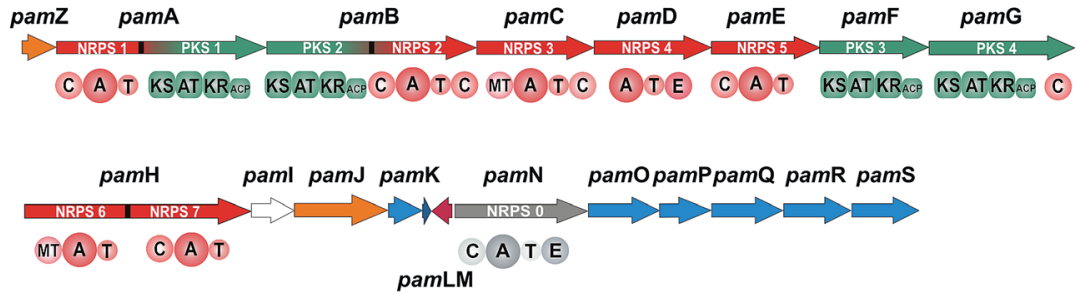

Fig. 4 NRPS and NRPS/PKS hybrid gene clusters harbored by P. larvae ERIC I and II. (A) The dhb gene cluster is harbored by both genotypes and responsible for the biosynthesis of the siderophore bacillibactin. (B) A monomodular NRPS-like gene cluster responsible for the biosynthesis of unknown compound(s). (C) The lipopeptide gene cluster of ERIC II is responsible for the biosynthesis of the paenilarvins. The genotype ERIC I harbors a significantly shorter form of the gene cluster; the corresponding secondary metabolite is not known yet. (D) The sev gene cluster of the genotype ERIC II is responsible for the biosynthesis of the tripeptide sevadicin. The homologue gene cluster of the genotype ERIC I is slightly bigger; the corresponding secondary metabolite is not identified yet. (E) The pam gene cluster is harbored by both genotypes and is responsible for the biosynthesis of the complex NRPS/PKS hybrid antibiotics paenilamicins. Genes responsible for precursor biosynthesis (blue), genes responsible for resistance/transport (orange), genes responsible for transcriptional regulation (red), genes of unknown function/hypothetical proteins (white). C: condensation domain; A: adenylation domain; T: thiolation domain; TE: thioesterase domain; TR: thioesterase reductase domain; AMT: class-III aminotransferase domain; E: epimerization domain; MT: methylation domain; KS: ketosynthase domain; AT: acyl transferase domain; KR: ketoreductase domain; ACP: acyl carrier protein.

(Fig. 3 and 4). ${ }^{9}$ One NRPS was suggested to have similarities to the biosynthetic machinery of the siderophore bacillibactin and one NRPS/PKS hybrid gene cluster was suggested to encode the biosynthesis of a lipopeptide from the iturin family. An entirely cryptic cluster was a trimodular NRPS gene cluster which showed no apparent similarity to known gene clusters of other bacteria. A complex NRPS/PKS hybrid gene cluster showed similarities to the zwittermicin and xenocoumacin gene clusters as well as to other gene clusters dedicated to be responsible for the biosynthesis of NRP prodrugs. We set out for a genetic and biochemical characterization of these gene clusters followed by analytical characterization of their biosynthetic products as basis for elucidating their biological role in AFB pathogenesis.

\section{Bacillibactin - occurrence of siderophores in P. larvae}

The gene cluster of $P$. larvae coding for the siderophore biosynthesis shows strong homology to the bacillibactin biosynthesis gene clusters of the Bacillus cereus sensu lato group and of Bacillus subtilis as well as to the paenibactin biosynthesis gene cluster of Paenibacillus elgii $69 .^{42}$ Likewise to the bacillibactin gene cluster the $P$. larvae gene cluster was named $d h b$ cluster (Fig. 4A). ${ }^{42}$ The $d h b$ cluster is about $11 \mathrm{kbp}$ in size and consists of five genes ( $d h b \mathrm{~A}, d h b \mathrm{C}, d h b \mathrm{E}, d h b \mathrm{~B}$, and $d h b \mathrm{~F}$ ), which were annotated to encode for a 2,3-dihydro-2,3-dihydroxybenzoate dehydrogenase (DhbA), an isochorismate 
synthase (DhbC), a DHB-AMP-ligase (DhbE), an isochorismatase (DhbB), and a dimodular peptide synthetase (DhbF), respectively. On the protein level DhbACEBF of $P$. larvae and DhbACEBF of $B$. cereus show a high similarity between 7079\%. Proteins DhbA, B and C are predicted to synthesize 2,3dihydroxybenzoate (DHB) from chorismate. The $60 \mathrm{kDa}$ DHBAMP-ligase DhbE with a similarity of $73 \%$ to $\mathrm{DhbE}$ of $B$. cereus is a stand-alone A domain, predicted to activate DHB. DhbF, with a similarity of $71 \%$ to $\mathrm{DhbF}$ of $B$. cereus, is a $267 \mathrm{kDa}$ dimodular NRPS. The specificity-conferring amino acid sequence (DILQVGLIWK) of the A-domain is identical to DhbF1 of B. subtilis which activates Gly. ${ }^{35}$ The second A domain (DFWNIGMVHK) is identical to DhbF2 of B. subtilis and activates Thr. ${ }^{35}$ Both NRPSs DhbF1 and DhbF2 show a typical arrangement with a condensation (C) domain, an adenylation (A) domain and a thiolation (T) domain. The biosynthesis works in an iterative manner of three cycles and in the final stage, the C-terminal thioesterase (TE) domain of DhbF2 performs cleavage of the final peptide from the NRPS machinery under cyclisation to the macrolacton. ${ }^{35}$ Initially bioinformatic studies suggested the $d h b$ cluster of $P$. larvae to encode for the NRPS machinery to synthesize paenibactin, a close bacillibactin homolog with Ala instead of Gly. ${ }^{39}$ For P. larvae strain DSM25430 (ERIC II) and strain DSM25719 (ERIC I) a combination of gene inactivation and mass spectrometric fragmentation analyses performed by our groups revealed that the NRPS machinery synthesizes the catechol-type siderophore bacillibactin and not paenibactin as expected before. ${ }^{42}$ Bacillibactin is the cyclic trimeric lactone of 2,3-dihydroxybenzoyl-Gly-L-Thr, ${ }^{35}$ which was assigned based on bioinformatics analysis and comparison with a reference compound from B. subtilis (Fig. 4). In general the dedicated function of siderophores is to secure iron supply under low iron

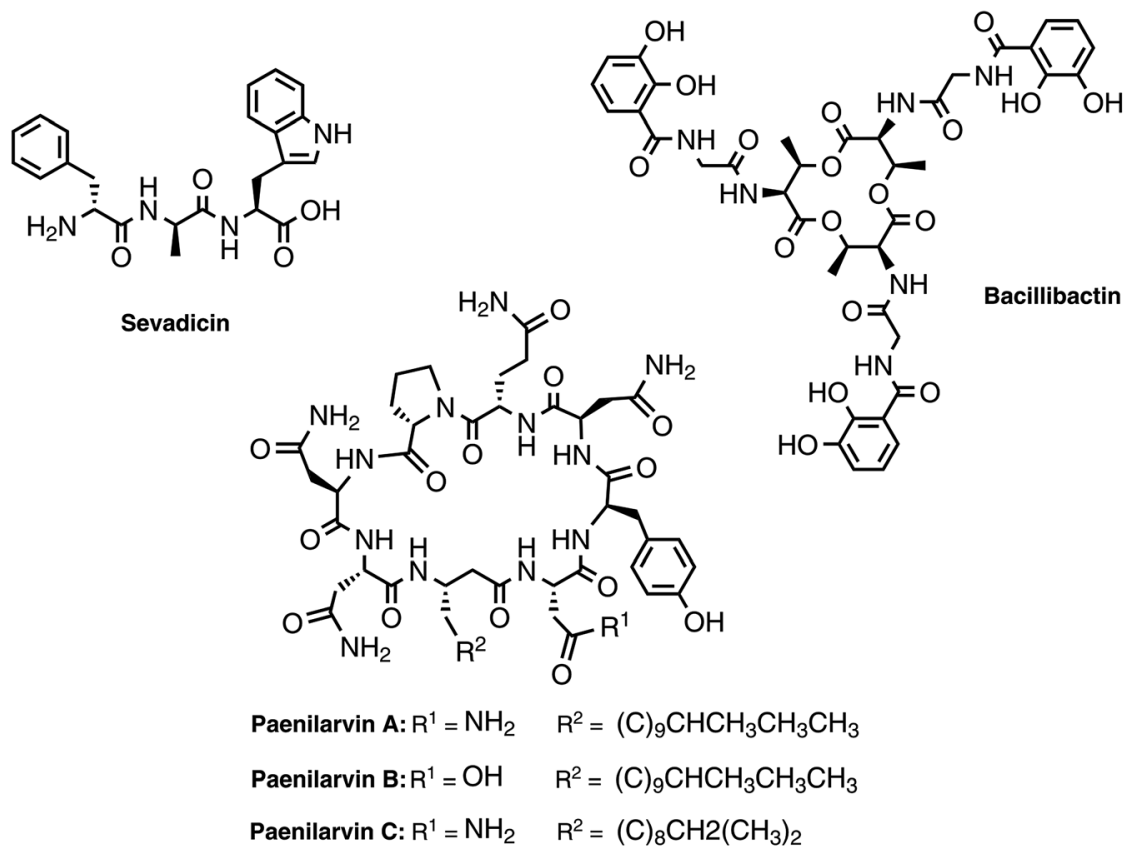<smiles>[R7]C(O)C[C@H](O)[C@@H](O)C(=O)N[C@@H](C)C(=O)N[C@@H](CNC)C(=O)N[C@@H]([R7])C(=O)N[C@H](CO)[C@H](O)C[C@H](O)CC(=O)N[C@@H](CNC)C(=O)NCC(=O)NCCCCNCCCN</smiles>

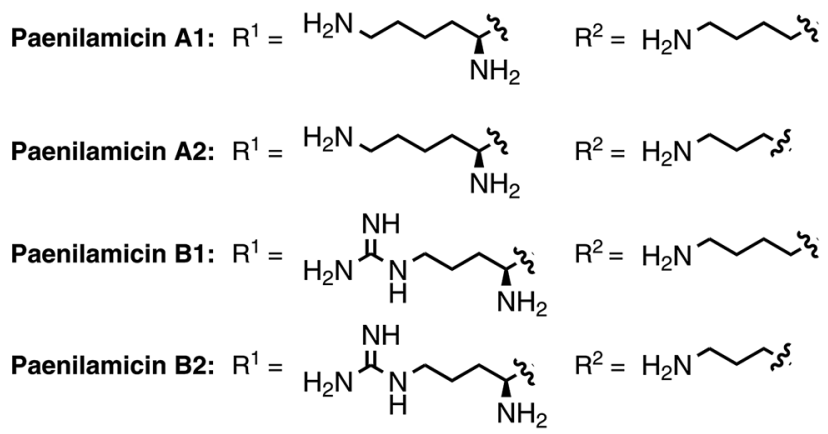

Fig. 5 Nonribosomally synthesized metabolites by $P$. larvae. 
conditions. ${ }^{\mathbf{4 3 4 4}}$ For most pathogenic bacteria iron is a limiting factor and both, pathogen and host are competing for iron. Therefore, many pathogens, but also non-pathogenic bacteria synthesize and secrete siderophores as $\mathrm{Fe}^{3+}$ scavengers in iron limiting conditions. Comparison of wild-type strains and the appropriate gene inactivation strains $(\Delta d h b \mathrm{~F})$ of both genotypes in larval infection assays however revealed no significant differences neither in total larval mortality nor in disease progression (cumulative larval mortality). ${ }^{42}$ Therefore, bacillibactin cannot be regarded as a virulence factor of $P$. larvae. In literature both is described, siderophores that act as virulence factors and siderophores not involved in pathogenicity. Recently, it could be shown that a B. cereus strain, unable to produce bacillibactin, showed an attenuated bacterial pathogenesis in insects. ${ }^{45}$ However, for a $B$. anthracis strain, insufficient in bacillibactin production, no phenotypical differences could be detected compared to the wild-type strain, suggesting that bacillibactin has no role in virulence. ${ }^{46}$

\section{Paenilarvins - iturin-like lipopeptide biosynthesis}

As mentioned above, genotype ERIC II encodes a 37 kbp hybrid PKS/NRPS gene cluster responsible for the biosynthesis of iturin-like lipopeptides, the paenilarvins (Fig. $4 \mathrm{~B}$ and 5). ${ }^{47}$ The iturins are structurally characterized by a macrocyclic heptapeptide core with an N-terminal $\beta$-amino fatty acid of various chain lengths. ${ }^{48}$ The $\beta$-amino group forms the macrolactam with the C-terminus of the heptapeptide. ERIC II contains one gene cluster with four ORFs of the same transcriptional directionality (Fig. 4B). The gene cluster arrangement is strongly reminiscent of the gene clusters responsible for the biosynthesis of the Bacillus lipopeptides iturin $\mathrm{A}$ and mycosubtilin. ${ }^{\mathbf{3 4 4 9}}$ The first gene (c18780) encodes a trans-acting acyltransferase, which shows 63\% sequence identity with ItuD from the iturin A biosynthesis. The initial PKS/NRPS encoded by c18770 shows $71 \%$ identity to the PKS/NRPS MycA of the mycosubtilin biosynthesis. Gene c18770 encodes the biosynthesis machinery for acylation and PKS-mediated extension of the acyl chain under involvement of the trans-acting acyltransferase and the reductive amination of the lipid chain by the class-III aminotransferase domain. Furthermore, the NRPS module for condensation of the first amino acid is encoded by c18770. Four additional A domains are found in c18760, whereas NRPS4 and 5 harbor epimerization domains for the stereochemical conversion of the activated L-amino acid to the cognate D-amino acid. On the protein level c18760 shows $72 \%$ identity with the NRPS MycB from Bacillus atrophaeus. Gene c18750 encodes for the NRPS responsible for the incorporation of the last two amino acids of the lipopeptide, whereas NRPS8 harbors an epimerization (E) domain. Additionally, the final NRPS contains a thioesterase domain, responsible for cyclisation and release of the lipopeptide.

The isolation and structure elucidation was achieved for three new iturin-family lipopeptides named paenilarvins accomplished from the $P$. larvae strain DSM25430. ${ }^{47}$ Four additional derivatives of the paenilarvins were identified present in low amounts, but were not further characterized. Mass spectrometric data led to suggest variations in chain length and stereochemistry in the $\beta$-amino fatty acid side chain. ${ }^{47}$ Paenilarvin A shows an identical amino acid sequence as the recently identified mojavensin A from Bacillus mojavensis B0621A, but differs in the $\beta$-amino fatty acid side chain (Fig. 5). ${ }^{50}$ In paenilarvin B, Asn at position 2 is exchanged by Asp (Fig. 4). Paenilarvin $\mathrm{C}$ is a paenilarvin A analogue shortened by two methylene groups in the $\beta$-amino fatty acid side chain (Fig. 5). The biosynthesis begins with the activation of a fatty acid, which is likely derived from the primary metabolism. Subsequently, the fatty acid is loaded onto the first $\mathrm{T}$ domain, followed by a chain extension with malonyl-CoA catalyzed by PKS module and the trans-acting acyltransferase (c18780). The action of the aminotransferase (AMT) domain converts the extended fatty acid into the $\beta$-amino fatty acid by reductive transamination. The $\beta$-amino fatty acid is then condensed with Asx, catalyzed by the condensation domain of the first NRPS module. The next four NRPS modules, all expressed by the same gene (c18760), catalyze the activation and condensation of Tyr, Asn, Gln and Pro, respectively. Hereby, the first two NRPS harbor epimerization domains, resulting in $\mathrm{D}$-Tyr and $\mathrm{D}$-Asn in the final peptide. The last gene of the cluster (c18750) encodes the final two NRPS, responsible for the activation and condensation of two Asn, whereas the first Asn is converted to D-Asn by the epimerization domain. The last domain of the c18750 gene product is a thioesterase domain, which catalyzes the cyclisation and release of the paenilarvins.

Most members of the iturin family lipopeptides show strong antifungal and cytolytic activity by disruption of the plasma membrane, whereas the fatty acid chain length seems to be important for the bioactivity. ${ }^{51,52}$ In contrast to their good antifungal properties, most iturin-type lipopeptides show no or only weak antibacterial activity. ${ }^{48}$ The same was observed for the paenilarvins: neither paenilarvin A, nor paenilarvin B showed antibacterial activity against Gram-positive or Gram-negative bacteria, but both compounds were active against various filamentous fungi and yeasts: Mucor hiemalis, Aspergillus clavatus, Botryotinia fuckeliana, Hormoconis resinae, Penicillium capsulatum, Saccharomyces cerevisiae, Rhodotorula glutinis, Candida albicans, Wickerhamomyces anomalus, Nematospora coryli, Trichosporon oleaginous, Debaryomyces hansenii and Pichia membranifaciens. Paenilarvin A was significantly more active ( $\mathrm{MIC}=$ 2.1-4.2 $\left.\mu \mathrm{g} \mathrm{mL}^{-1}\right)$ than Paenilarvin $\mathrm{B}\left(\mathrm{MIC}=8.3-33.3 \mu \mathrm{g} \mathrm{mL}{ }^{-1}\right)$. In addition to the antimicrobial assays, the paenilarvins were also tested in a cell culture assay against the mouse fibroblast cell line L929. Thereby, paenilarvin A and B both showed also significant cytotoxicity (paenilarvin $\mathrm{A} \mathrm{IC}_{50}=4 \mu \mathrm{g} \mathrm{mL} \mathrm{m}^{-1}$; paenilarvin $\left.\mathrm{B}: \mathrm{IC}_{50}>10 \mu \mathrm{g} \mathrm{mL}{ }^{-1}\right)$. The authors were further interested in the effects of paenilarvin A and B against bee larva. Therefore, bee larvae were fed with paenilarvin A or B $(5 \mu \mathrm{g})$ at days 4, 5 and 6 and larval mortality was recorded for 22 days. For both compounds a significant activity against bee larvae could be demonstrated: for paenilarvin A the mortality was $25 \%$ higher and for paenilarvin B 35\% higher compared to the larval control group. In this study, paenilarvin $\mathrm{C}$ was not tested in any 
of the assays, whereas the effects of the shortened lipid chain for the increased bioactivity seem interesting. The authors estimate their studies on bee larvae as preliminary data, which have to be confirmed with different concentrations of compounds and with larvae at different developmental stages. The question whether paenilarvins are required by $P$. larvae to fight fungi as ecological niche competitors or to kill bee larva could not be answered in this study. The authors discussed a further hypothesis for the role of the paenilarvins: at subinhibitory concentrations they could act as signaling molecules, which is a hypothesis already suggested previously for other systems. ${ }^{53,54}$ In contrast, the corresponding gene cluster from $P$. larvae DSM25719 (genotype ERIC I) is much smaller $(\sim 12.5 \mathrm{kbp})$ and contains only two genes (Fig. 4B). In this context only the biosynthetic machinery for the acylation, PKS-mediated extension and amidation as well as the NRPS for the incorporation of the first amino acid are present (Fig. 4B). The NRPS shows homology to NRPS9 of ERIC II, encoded by gene c18750. Accordingly, the ERIC I gene cluster could be responsible for the biosynthesis of another not yet identified (lipo)peptide or might be even inactive. However, no studies on the ERIC I gene cluster were published yet.

\section{Sevadicin - A nonribosomal tripeptide}

The genome of strain DSM25430 (genotype ERIC II) contains an $11.7 \mathrm{kbp}$ gene cluster ( $\mathrm{eev}$ cluster) consisting of only two genes (sevA and $s e v \mathrm{~B}$ ) and coding for three NRPS modules without the presence of genes coding for tailoring enzymes or transport (Fig. 4C). In the NRPS genes epimerization (E) domains that catalyze the inversion of the $\alpha$-stereocenter of amino acids are located in the first two modules while a TE domain was located at the C-terminal part of module 3. The predictions for A domain specificities were ambiguous, such that sequence details as well as peptide size were elusive. Help came from gene inactivation strains, incapable of sevadicin production. Subsequent analytical characterization rendered a linear peptide, called sevadicin with the sequence D-Phe-D-Ala-Trp (Fig. 5). ${ }^{55}$ While for strain DSM25430 (ERIC II) a peptide could be identified, strain DSM25719 (ERIC I) contains an NRPS gene cluster of five genes with four NRPS modules (Fig. 2C). The product of gene c02110 is annotated as a lone-standing A domain, predicted to activate Cys. At the protein level SevA and the product of c02090 are highly homologous, whereas the ERIC I NRPS is slightly shorter (927 aa) compared to SevA (1080 aa). Comparison of the ten highly conserved core motifs ${ }^{56}$ of the adenylation domains revealed that the ERIC I A domain lack the core motifs A1 and A2, suggesting an inactive module. Hereby, the involvement of the lone-standing A domain (gene c02110) as a trans-acting A domain could be hypothesized. Due to our inspection, the NRPS module encoded by gene c02100 seems to be active and is predicted to activate Val. The NRPS module encoded by c02120 shows a high homology to NRPS2 of SevB, with the exception of the first $\mathrm{C}$ domain. However, the $\mathrm{C}$ terminal $\mathrm{C}$ domain is homologous to the $\mathrm{C}$ domain of NRPS3 of SevB. NRPS4 (encoded by c02130) is identical to the A-T-TE domains of SevB. Nevertheless, until to date no peptide was identified in ERIC I secretome.

Testing the secretome of the sev gene inactivation strain showed that the antibacterial activity was clearly decreased while not completely lost compared to the wild-type secretome. Synthetic sevadicin showed only weak antibacterial activity against $B$. megaterium, suggesting alternative functions of sevadicin. These may be signaling networks, as it has recently shown for the aureusimines: nonribosomally synthesized diketopiperazine-like compounds produced by Staphylococcus aureus, ${ }^{57}$ which might be involved in regulating the expression of genes associated with virulence and with regulatory and redox-associated genes, ${ }^{58}$ suggesting a potential function for regulating a metabolic switch of $S$. aureus. Interestingly, $P$. larvae previously has also been hypothesized to undergo a metabolic switch during pathogenesis. ${ }^{16}$ In the larval gut during the non-invasive phase, $P$. larvae mainly takes its nutrients from the larval food ingested by larvae. ${ }^{18}$ Subsequently during the invasive phase, $P$. larvae switches to consuming larval structures, first the peritrophic membrane protecting the gut, ${ }^{17,19}$ then totally decomposing all larval tissues yielding a ropy mass consisting of a pure P. larvae culture.

\section{Paenilamicins - complex peptide- polyketide hybrids}

A remarkably complex NRPS/PKS hybrid gene cluster (pam gene cluster) of about $60 \mathrm{kbp}$, which shows no greater similarities to any known gene clusters, is found in both genotypes ERIC I and ERIC II, respectively (Fig. 4D). The biggest part of the gene cluster consists of five NRPS (pam C-E, $\operatorname{pam} \mathrm{H}$ and $\operatorname{pam} \mathrm{N}$ ), two PKS (pamF and G) and two PKS/NRPS hybrid genes (pamA and B).$^{59}$ Six genes are involved in precursor biosynthesis (pam $\mathrm{K}$ and pamO-S). Two genes have a likely function in transport and/or resistance (pamZ and pamJ). PamM is most probably responsible for transcriptional regulation, whereas PamL is a lonestanding acyl-carrier protein (ACP) and PamI shows weak homology to BtrH-like proteins.

The considerably challenging structure elucidation was achieved by isolating four derivatives, named paenilamicins (Fig. 4). ${ }^{60}$ Based on the structures of paenilamicin A1/A2 and paenilamicin B1/B2 a comprehensive biosynthetic picture could be drawn (Fig. 6A): the A domain of PamA shows relaxed substrate specificity and must be capable of activating either Lys or Arg. The biosynthesis continues with a PKS1-mediated Claisen condensation with malonyl-CoA and a ketoreductasecatalyzed reduction of the amino acid carbonyl to yield a secondary alcohol and the generation of a cadaverine (Cad) or agmatine (Agm) residue, respectively. A subsequent Claisen condensation with an unusual $(2 R)$-hydroxymalonyl is then performed by the PKS part of the PKS/NRPS hybrid PamB, followed by another ketoreduction. $(2 R)$-Hydroxymalonyl-ACP is most likely provided by the catalytic action of PamKOPQ, proteins that show high similarity to $(2 R)$-hydroxymalonyl-ACP biosynthesis proteins known from the zwittermicin biosynthesis. ${ }^{\mathbf{6 1 , 6 2}}$ PamK shows 78\% similarities to the 3-hydroxyacyl- 
A)

A) Lys or Arg

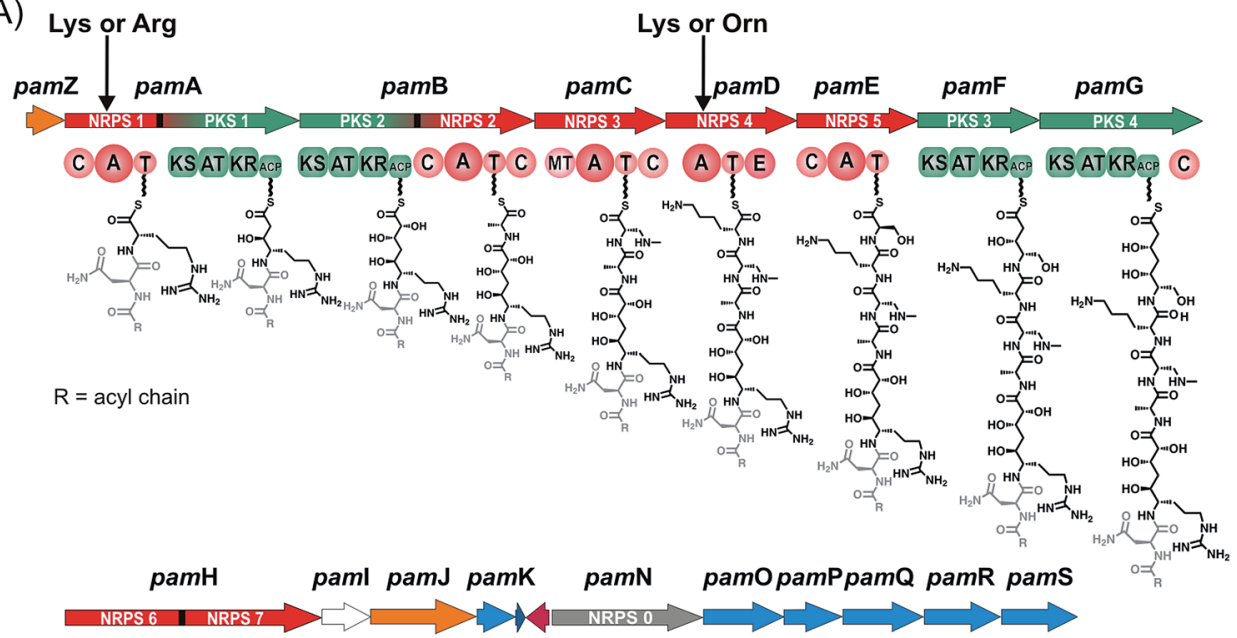

MT A. C A T

C A T E
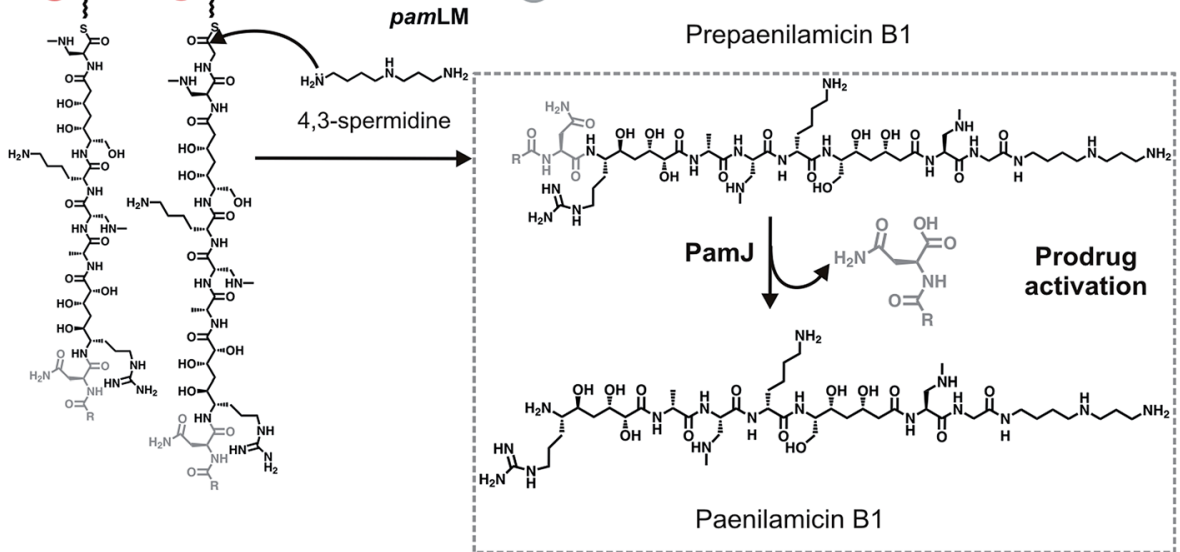

B)

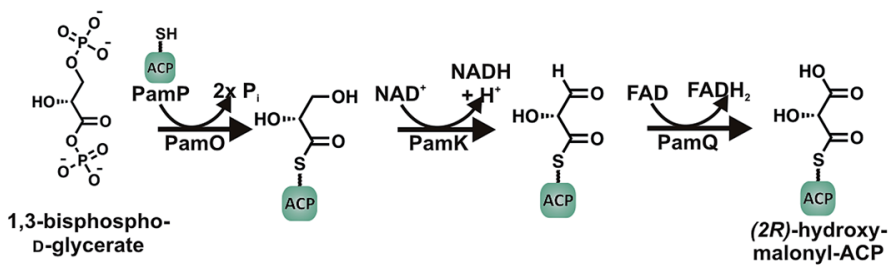

C)

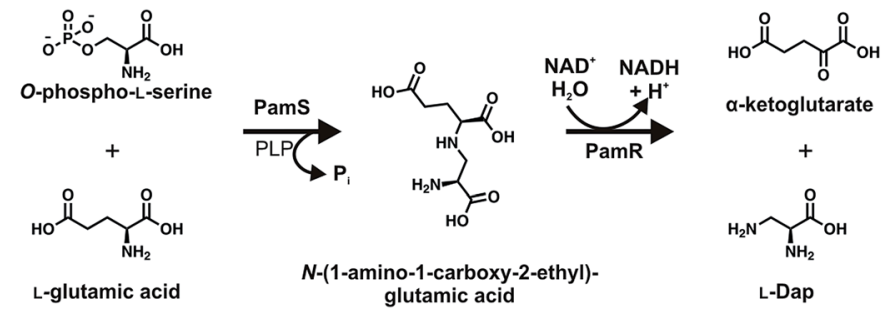

Fig. 6 The pam gene cluster from P. larvae DSM25430 and proposed biosynthesis of paenilamicins and precursors. (A) The proposed assembly line of prepaenilamicin B1 and its activation to paenilamicin B1. The prepaenilamicin B1 assembly line starts presumably with the activation of acyl-D-Asn (grey; R = fatty acid) by NRPSO (pamN). Prepaenilamicin B1 is released in a TE-independent fashion by the nucleophilic condensation of 4,3-spermidine to Gly. Prepaenilamicin is potentially activated by the transmembrane transporter/peptidase PamJ (grey boxed). Genes responsible for precursor biosynthesis (dark-blue), genes responsible for resistance/transport (orange), genes responsible for transcriptional regulation (red), genes of unknown function/hypothetical proteins (white). (B) Putative (2R)-hydroxymalonyl-ACP biosynthesis with the glycolysis intermediate 1,3-bisphospho-D-glycerate as substrate. (C) L-Dap biosynthesis from O-phospho-L-Ser and L-Glu. C: condensation domain; A: adenylation domain; T: thiolation domain; E: epimerization domain; MT: methylation domain; KS: ketosynthase domain; AT: acyl transferase domain; KR: ketoreductase domain; ACP: acyl carrier protein; PLP: pyridoxal 5'-phosphate. 
CoA-dehydrogenease (ZmaG), PamO shares 88\% similarities with the FkbH-like protein ( $\mathrm{ZmaN})$ and PamP is annotated as an ACP with high similarity (86\%) to $\mathrm{ZmaD} / \mathrm{H}$, whereas PamQ is an acyl-CoA dehydrogenase with $89 \%$ similarity to ZmaE. The proposed biosynthesis of $(2 R)$-hydroxymalonyl-ACP requires glycolysis intermediate 1,3-bisphospho-D-glycerate as a substrate (Fig. 6B) ${ }^{61}$ The 1,3-bisphospho-D-glycerate is tethered to PamP and dephosphorylated by PamO. The glycerate-ACP is subsequently oxidized by PamK and PamQ, respectively yielding in (2R)-hydroxymalonyl-ACP (Fig. 6B). Next, a D-Ala is condensed to the building block by NRPS2. Hereby, D-Ala is not stereochemically converted by an epimerization domain of NRPS2 (no $\mathrm{E}$ domain could be detected by sequence analysis). Because of the occurrence of a gene coding for an alanine racemase it is proposed that D-Ala is provided by this enzyme and directly activated by the A domain, as it was shown for cyclosporin and HC toxin. ${ }^{\mathbf{6 3} 64}$ The biosynthesis continues with the condensation of the amino acids $N$-methyl-diaminopropionic acid (mDap), DLys/D-Orn, and Ser. Thereby, Dap is provided by Pams and PamR from the substrates $O$-phospho-L-Ser and Glu (Fig. 6C). ${ }^{65}$ PamS is a 2,3-diaminopropionate biosynthesis protein (SbnBlike, $80 \%$ similarity) and PamR is a pyridoxal-5'-phosphatedependent protein subunit $\beta$ (SbnA-like, 80\% similarity), which are known from the Dap biosynthesis of the staphyloferrin B biosynthesis (Fig. 6C) ${ }^{65}$ The presence of $N$-methyl-Dap is supported by an $N$-methylation domain in the NRPS PamC. In contrast to common $\mathrm{N}$-methylations occurring at the peptide bond, this $N$-methylation is located in the amino acid sidechain. As already described for the A domain of PamA, the A domain of PamD shows also a relaxed substrate specificity and is capable to activate Lys and Orn, resulting in another two paenilamicin derivatives. The biosynthesis proceeds with two consecutive condensations of malonyl-CoA with followed ketoreductions catalyzed by PKS3 (PamF) and 4 (PamG). Together, PamE-G proteins are responsible for the biosynthesis of the structural motif galantinic acid (Gla). Interestingly, the same structural motif is found in the siderophor anachelin produced by the cyanobacterium Anabaena cylindrical, suggesting the same biosynthetic setup. ${ }^{66}$ Catalyzed by the bimodular NRPS PamH, another mDap and a Gly are attached. An interesting hypothesis is provided for the attachment of the C-terminal 4,3-spermidine. The protein PamI shows homology to the BtrH protein family. A functional classification for a BtrH protein was given for the biosynthesis of butirosin, where the $\mathrm{BtrH}$ protein is described as an aminoglycoside 1- $\mathrm{N}$-acyltransferase responsible for the ligation of the aminoglycoside side chain of butirosin. ${ }^{67}$ At the C-terminus of PamH (after the T domain of the Gly specific NRPS) a similar domain could be identified and the authors hypothesized that the domains could somehow be responsible for the directional attachment of 4,3spermidine to the paenilamicins and thereby release of the peptide from the NRPS.

Another interesting aspect is the suggested production of a prodrug called prepaenilamicin, which parallels the (pre)xenocoumacins, compounds from Xenorhabdus nematophila described by Bode and coworkers. ${ }^{68,69}$ Therein two genes seem to be conserved: a NRPS specific for the activation of (acyl)-D-Asn or (acyl)-D-Gln and a D-Asn specific carboxypeptidase. For the pam system the A domain of NRPS8 (PamN) is predicted to activate Asn or Asx by three different prediction tools and harbors an E domain responsible for transformation of L-Asx into D-Asx. Moreover, PamN is homologous to the starting module XcnA of the xenocoumacin biosynthesis gene cluster as well as to starting modules of other biosynthesis gene clusters responsible for the biosynthesis of nonribosomal prodrugs like amicoumacin (B. pumilus), zwittermicin (B. thuringiensis), colibactin (E. coli), and other similar NRPS/PKS clusters with unknown products that all are predicted to activate Asn or Asx. ${ }^{68,69}$

Bode and coworkers proposed two different architectures of the peptidases: type I with XcnG as the model peptidase comprises a signal peptide followed by a periplasmatic D-Asn specific carboxypeptidase and three C-terminal transmembrane helices. Type II has a signal peptide followed by a peptidase domain, nine transmembrane helices and an $\mathrm{ABC}$ transporter. ${ }^{68}$ The peptidase subunit is responsible for the specific cleavage of the acylated D-Asp moiety of the prexenocoumacins, resulting in the bioactive xenocoumacins. ${ }^{68}$ The protein PamJ was found to be homologous to XcnG suggesting that PamJ could be a peptidase (Fig. 6A). Although PamJ shows structural differences (type II architecture) to XcnG (type I architecture) a comparable biosynthesis and pro-drug activation mechanism seems to be likely (Fig. 6A). The described pro-drug mechanism is a strategy of the producer strain to avoid self-damaging.

For the paenilamicins antibacterial activity against various Gram-positive strains (P. alvei, B. subtilis, B. licheniformis, and B. megaterium) was found, which are all strains with a potential association with honey bees. ${ }^{59}$ Beside the antibacterial activity the paenilamicins exhibit also antifungal activity against yeasts ( $P$. pastoris and $S$. cerevisiae) and the plant pathogenic filamentous fungus $F$. oxysporum. ${ }^{59}$ Cytotoxic effects found in vitro against the Trichoplusia ni (Lepidotera-derived) insect cell line Tn5, suggested that paenilamicin could act as a toxin against bee larvae. However bee larva infection assays with a $P$. larvae strain unable to synthesize paenilamicin (P. larvae $\Delta$ pamA) showed no significant differences in total mortality of bee larvae. ${ }^{59}$ These results show that in the $P$. larvae/honey bee larvae system it is obviously not always possible to infer from in vitro data on in vivo function. Nevertheless, the authors also analyzed the time course of infection revealing that the curve for cumulative mortality was shifted for the larval groups infected with the mutant strain $(\Delta p a m \mathrm{~A})$, indicating a significantly delayed onset of mortality in comparison to the groups infected with wild-type $P$. larvae. Accordingly, the $\mathrm{LT}_{100}$ (lethal time) also significantly differed: wild-type $P$. larvae required about 8 days, whereas the mutant strain needed 12 days to accomplish killing of all infected larvae. ${ }^{59}$ The proposed main function of paenilamicin was shown in a recently published study: ${ }^{60}$ in the corresponding assay larvae were co-infected with both $P$. larvae and the saprophytic bacterium $P$. alvei. After larval death dead larvae were examined for $P$. alvei survival by determination of $P$. alvei growth on agar medium. This assay showed that only $11 \%$ vital $P$. alvei could be recovered from larvae infected with wild-type $P$. larvae. Interestingly, in co-infection assays where larvae were 
infected with $P$. alvei and $P$. larvae $\triangle$ pamA the recovery rate of $P$. alvei was significantly higher (about $62 \%$ ). The results of the larvae co-infection assays show, that a main function of the paenilamicins is outcompeting competitors in the ecological niche larval gut and may explain why the ropy mass that remains after larval death is a pure culture of P. larvae (Fig. 2A).

\section{A cryptic monomodular NRPS-like gene cluster}

The genotypes, ERIC I (gene c22070) and ERIC II (gene c04880) both contain a monomodular NRPS-like gene (Fig. 4E). It encodes for an A domain, a $\mathrm{T}$ domain and a C-terminal thioesterase reduction (TR) domain, but lacks a $\mathrm{C}$ domain. The $\mathrm{A}$ domain of the $P$. larvae NRPS-like protein is predicted to activate Phe and the module arrangement has similarities to the fungal NRPS LnaA (1042 aa) and LnbA (1007 aa) from Aspergillus flavus NRRL3357, ${ }^{70}$ although the putative NRPS-like protein from P. larvae (1239 aa) shares only weak homology to LnaA and LnbA, respectively. The A domains of LnaA and LnbA specifically activate Tyr and biosynthesis products are piperazine and pyrazine derivatives, as well as a hemiacetal-containing morpholine. ${ }^{70}$ In a biosynthesis model the authors suggest that Tyr is activated by the A domains of LnaA and/or LnbA, transferred to the $\mathrm{T}$ domains and subsequently reduced to yield a tyrosinal by the catalytic action of the TR domain. Further reduction and cyclisation is supposed to be catalyzed be the reductases LnaB and LnbB. ${ }^{70}$ In the context of the P. larvae cluster no additional reductases were identified, furthermore despite the NRPS gene no further genes show homologies to the $\ln a$ or $\ln b$ cluster. The structures are reminiscent of aureusimines from $S$. aureus and diketopiperazines from Streptomyces although different biosynthesis mechanisms as bimodular NRPS and tRNAdependent assembly are employed. ${ }^{57,71}$ Likewise to above mentioned bacteria, the diketopiperazines and the piperazine/ pyrazine might play a role in a signaling networks. ${ }^{57,70,71}$ From the present data we hypothesize that $P$. larvae is able to produce related compounds which could have a similar function as quorum sensing molecules, which needs to be proven in further work.

\section{Gene clusters of ribosomally synthesized secondary metabolites}

Apart from the above mentioned gene clusters encoding for nonribosomal peptides, the genotypes ERIC I and ERIC II contain also genes encoding for the synthesis of ribosomal peptides. The genomes of $P$. larvae strains DSM25430 and DSM25719 were analyzed with the antiSMASH ${ }^{72}$ online tool to assess the potential for the biosynthesis of bacteriocins and ribosomally synthesized and posttranslationally modified peptides (RiPPs).

The manual assignment of genes rendered three putative bacteriocin and RiPP gene clusters for genotype ERIC II, thereof one lanthipeptide and two bacteriocins. For genotype ERIC I putative biosynthesis genes or gene cluster-like arrangements for eight bacteriocins and one lanthipeptide were found. In ERIC II the lanthipeptide cluster comprises a gene (c31290) coding for a putative lantibiotic dehydratase with a C-terminal thiopeptide-type bacteriocin biosynthesis domain. Gene c31300 encodes a LanC-like cyclase and c31310 for a truncated ABC transporter (183 aa). A protease was not found in the cluster, but is also not universally found in class I gene clusters, ${ }^{73,74}$ while however the assigned prepropeptide gene (c31270) has only weak sequence homology to known prepropeptides. A putative gene cluster for the biosynthesis of a bacteriocin and with homology to gene clusters of several B. amyloliquefaciens and $B$. subtilis species is gene c36580. NCBI's conserved domain search $^{75}$ showed that the C-terminal part has homologies to class IId cyclic uberolysin-like bacteriocins. ${ }^{7}$ A putative ABC transporter of 233 aa (gene c36600), could be responsible for transport and self-resistance. Even if a large part of the genes of this cluster could not be assigned regarding their functions one could assume a functional gene cluster for the expression of a bacteriocin. Another uberolysin-like putative bacteriocin (73 aa) is encoded by gene c 40350 with $49.3 \%$ identity and $69.9 \%$ similarity to uberolysin from Streptococcus uberis. ${ }^{77}$ The truncated gene product of c40340 (191 aa) shows only weak homology to the cyclizing protein UblB (535 aa), ${ }^{77}$ assuming that the P. larvae protein is non-functional. Three putative $\mathrm{ABC}$ transporters are encoded by the genes c40280, c40250 and c40240.

Probably an incomplete class II lanthipeptide gene cluster is spanning the region 1806 134-1 812604 , which contains a truncated lanM-like gene and a putative prepropeptide (c19180) with weak homology to known lanthipeptides and lacking characteristic features of precursor peptides. Likewise, bacteriocin gene clusters (c05230, c05830, c21080, c21140, c24550, c28660, c31740, c34160) putatively are disrupted or incomplete.

\section{Summary and outlook}

Genome sequencing of Paenibacillus larvae strains DSM25430 (genotype ERIC II) and DSM25719 (genotype ERIC I) revealed the presence of gene clusters coding for the synthesis of secondary metabolites. With a size of 4056006 bp (ERIC II) and 4579589 bp (ERIC I), $2.99 \%$ and $2.23 \%$ of the genomes are devoted to the biosynthesis of small molecules, respectively (Fig. 3).

The gene clusters encode NRPS peptides and NRPS/PKS hybrid peptides of which four structural families have been identified (Fig. 4 and 5). Interestingly, exclusive polyketide synthases have not been identified. Furthermore, no gene clusters responsible for the biosynthesis of terpenes, aminoglycosides and other small molecules have been identified (own analysis). Putative clusters responsible for ribosomally synthesized peptides could be detected in both $P$. larvae genotypes, whereas antiSMASH analysis of genotype ERIC I showed more putative clusters (one lanthipeptide and eight bacteriocin clusters) compared to genotype ERIC II (one lanthipeptide, two bacteriocin clusters). Most clusters seem to be incomplete or exhibited only weak homologies to known gene clusters of ribosomally synthesized peptides. ${ }^{76,78}$ Nevertheless, some core precursor peptides showed homologies to known bacteriocins. ${ }^{79}$ 
Experimental data is necessary to prove which clusters are active and to elucidate the roles of bacteriocins and RiPPs in AFB.

In recent work four of the dedicated nonribosomal secondary metabolites were identified and their structures characterized. The siderophore bacillibactin is generally widespread among Bacillus species. Since gene inactivation studies of the gene cluster rendered no effect on virulence we hypothesize that the siderophore may be an evolutionary remnant still enabling $P$. larvae to sequester iron under certain circumstances. The other three compounds apparently have antimicrobial defense tasks: the nonribosomal tripeptide sevadicin shows antibacterial activity while paenilarvins, iturin-like lipopeptides, have antifungal activity against various filamentous fungi and yeasts. For iturin family lipopeptides fungicidal and strong cytolytic activity has been described while they display no or only weak antibacterial activities. ${ }^{\mathbf{4 8 5 1 , 5 2}}$ Finally, paenilamicins show both, antibacterial as well as antifungal activity in addition to cytotoxic activity against insect cells. ${ }^{59}$ It is very likely, that the array of these compounds acts in a synergistic manner as this was already described for effects on pathogenic fungi by bacillomycin and fengycin. ${ }^{\mathbf{8 0}}$ Exposure bioassays provided first evidence for the in vivo function of the paenilamicins during AFB pathogenesis: (i) by killing bacterial (and maybe fungal) competitors the paenilamicins enable $P$. larvae to fight its ecological niche and (ii) the paenilamicins influence the time-course of disease in bee larvae although the mechanism remains elusive. ${ }^{59}$ Therefore, paenilamicins act as virulence factors for $P$. larvae during larval infection. The high specialization of $P$. larvae on honey bees and the uniqueness of sevadicin and paenilamicin in this bacterium may indicate a more intricate role of these two compounds.

Still not clear is the cytotoxicity of compounds which becomes critical in the moment when $P$. larvae breaks through the midgut lumen. While chitinases and maybe also other enzymes dissolve cellular structures, secondary metabolites could have a function in protecting the larval cadaver against other bacteria and fungi that act as nutrient competitors. Another function, especially for sevadicin due to weak antibacterial activity, could be a role as a signaling molecule involved in the regulation of biological processes, as it could be shown for the aureusimines ${ }^{57,58}$ and other antibiotics. ${ }^{54}$

The described genomic differences between $P$. larvae ERIC I and ERIC II also concern the secondary metabolite gene clusters. The genome of the P. larvae genotype ERIC I harbors also the paenilamicin and the bacillibactin clusters, although paenilamicin could not be identified from fractionated secretomes yet. Additionally, ERIC I harbors two clusters that differ from homologous clusters from genotype ERIC II, and whose products are not identified yet. One of these clusters is homologous to the iturin gene cluster, which consists in contrast to the gene cluster of ERIC II, of only two genes. One of these genes is a trans-acting acyltransferase nearly identical to c18780 from ERIC II Ser103Ala; the other gene is a NRPS/PKS hybrid with high homology to gene c18770 from ERIC II, but harboring a TE domain at the C-terminus. This cluster could be responsible for the synthesis of another not yet identified (lipo)peptide or might be even an inactive gene cluster. Another gene cluster responsible for the biosynthesis of an unknown peptide from the genotype ERIC I is a homolog of the sev gene cluster. The ERIC I gene cluster consists of five genes (two genes in ERIC II) and likely includes a trans-acting A domain in the peptide synthesis. Close inspection of the gene cluster led us to suggest the synthesis of a tetrapeptide. Furthermore, a monomodular NRPS-like gene is harbored by both genotypes, whose compounds are not identified yet. NRPS-like proteins with the architecture A-T-TR are predominantly known from fungi. Such as the NRPS-like proteins from the fungus A. flavus, which are responsible for the biosynthesis of metabolites involved in a signaling network resulting in a metabolic switch. Interestingly, also $P$. larvae is undergoing a metabolic switch. ${ }^{16}$ Further work is necessary to identify all compounds produced by $P$. larvae and to illuminate their functions.

In conclusion entomopathogens appear as a rich and yet underinvestigated source of novel and structurally diverse secondary metabolites. The bee pathogen $P$. larvae is no exception to this rule and an in-depth study and characterization of the secondary metabolites produced by the honey bee pathogen $P$. larvae is highly relevant not only for bee pathologists but for a broader community interested in discovering and elucidating novel antibacterial substances. Hence, improving our knowledge of the secondary metabolites of $P$. larvae will allow a better understanding of roles of secondary metabolites in cell biology and pathogenesis, especially in AFB. In addition, the further characterization of novel metabolites, such as paenilamicin, could bring new resources in the drug development market.

\section{Acknowledgements}

The authors are grateful to the Ministries for Agriculture from Brandenburg and Sachsen-Anhalt, Germany, the German Research Foundation (Graduate School 1121), and the Cluster of Excellence "Unifying Concepts in Catalysis" (UniCat) coordinated by TU Berlin.

\section{Notes and references}

1 R. Morse and N. Calderone, Bee Cult., 2000, 128, 1-15.

2 M. A. Aizen, L. A. Garibaldi, S. A. Cunningham and A. M. Klein, Curr. Biol., 2008, 18, 1572-1575.

3 M. A. Aizen and L. D. Harder, Curr. Biol., 2009, 19, 915-918. 4 E. Genersch, Appl. Microbiol. Biotechnol., 2010, 87, 87-97.

5 E. Genersch, E. Forsgren, J. Pentikainen, A. Ashiralieva, S. Rauch, J. Kilwinski, I. Fries and J. Pentikäinen, Int. J. Syst. Evol. Microbiol., 2006, 56, 501-511.

$6 \mathrm{~J}$. Versalovic and M. Schneider, Methods Mol. Cell. Biol., 1994, 5, 25-40.

7 B. J. Morrissey, T. Helgason, L. Poppinga, A. Fünfhaus, E. Genersch and G. E. Budge, Environ. Microbiol., 2014, 17, 1414-1424.

8 S. Rauch, A. Ashiralieva, K. Hedtke and E. Genersch, Appl. Environ. Microbiol., 2009, 75, 3344-3347.

9 M. Djukic, E. Brzuszkiewicz, A. Fünfhaus, J. Voss, K. Gollnow, L. Poppinga, H. Liesegang, E. Garcia-Gonzalez, E. Genersch and R. Daniel, PLoS One, 2014, 9, e90914. 
10 E. Genersch, J. Invertebr. Pathol., 2010, 103, S10-S19.

11 E. Genersch, A. Ashiralieva and I. Fries, Appl. Environ. Microbiol., 2005, 71, 7551-7555.

12 N. Wilson-Rich, M. Spivak, N. H. Fefferman and P. T. Starks, Annu. Rev. Entomol., 2009, 54, 405-423.

13 L. Hasemann, Am. Bee J., 1961, 101, 298-299.

14 J. D. Hitchcock, A. Stoner, W. T. Wilson and D. M. Menapace, J. Kans. Entomol. Soc., 1979, 52, 238-246.

15 W. T. Wilson, J. Invertebr. Pathol., 1971, 17, 247-255.

16 D. Yue, M. Nordhoff, L. H. Wieler and E. Genersch, Environ. Microbiol., 2008, 10, 1612-1620.

17 E. Garcia-Gonzalez and E. Genersch, Environ. Microbiol., 2013, 15, 2894-2901.

18 S. Neuendorf, K. Hedtke, G. Tangen and E. Genersch, Microbiology, 2004, 150, 2381-2390.

19 E. Garcia-Gonzalez, L. Poppinga, A. Fünfhaus, G. Hertlein, K. Hedtke, A. Jakubowska and E. Genersch, PLoS Pathog., 2014, 10, e1004284.

20 A. Fünfhaus, A. Ashiralieva, R. Borriss, E. Genersch and A. Funfhaus, Environ. Microbiol. Rep., 2009, 1, 240-250.

21 A. Fünfhaus, L. Poppinga and E. Genersch, Environ. Microbiol., 2013, 15, 2951-2965.

22 P. O. Falnes and K. Sandvig, Curr. Opin. Cell Biol., 2000, 12, 407-413.

23 N. C. Simon, K. Aktories and J. T. Barbieri, Nat. Rev. Microbiol., 2014, 12, 599-611.

24 I. Carpusca, T. Jank and K. Aktories, Mol. Microbiol., 2006, 62, 621-630.

25 L. Poppinga, B. Janesch, A. Fünfhaus, G. Sekot, E. GarciaGonzalez, G. Hertlein, K. Hedtke, C. Schäffer and E. Genersch, PLoS Pathog., 2012, 8, e1002716.

26 A. Fünfhaus and E. Genersch, Environ. Microbiol. Rep., 2012, 4, 194-202.

27 E. C. Holst, Science, 1945, 102, 593-594.

28 G. F. White, USDA Bur. Entomol. Tech. Ser., 1906, 14, 1-50.

29 S. Vojvodic, S. M. Rehan and K. E. Anderson, PLoS One, 2013, 8, e72106.

30 C. Ash, J. A. Farrow, M. Dorsch, E. Stackebrandt and M. D. Collins, Int. J. Syst. Bacteriol., 1991, 41, 343-346.

31 C. Ash, F. G. Priest and M. D. Collins, Antonie van Leeuwenhoek, 1993, 64, 253-260.

32 T. Stein, Mol. Microbiol., 2005, 56, 845-857.

33 F. Lipmann, W. Gevers, H. Kleinkauf and R. Roskoski, Adv. Enzymol. Relat. Areas Mol. Biol., 1971, 35, 1-34.

34 E. H. Duitman, L. W. Hamoen, M. Rembold, G. Venema, H. Seitz, W. Saenger, F. Bernhard, R. Reinhardt, M. Schmidt, C. Ullrich, T. Stein, F. Leenders and J. Vater, Proc. Natl. Acad. Sci. U. S. A., 1999, 96, 13294-13299.

35 J. J. May, T. M. Wendrich and M. A. Marahiel, J. Biol. Chem., 2001, 276, 7209-7217.

36 K. Arima, A. Kakinuma and G. Tamura, Biochem. Biophys. Res. Commun., 1968, 31, 488-494.

37 J. R. Cath, T. S. G. Jones and S. Wilkinson, Ann. N. Y. Acad. Sci., 1949, 51, 917-923.

38 Y. Kajimura and M. Kaneda, J. Antibiot., 1996, 49, 129-135.

39 Y. Wen, X. Wu, Y. Teng, C. Qian, Z. Zhan, Y. Zhao and O. Li, Environ. Microbiol., 2011, 13, 2726-2737.
40 X.-C. Wu, C.-D. Qian, H.-H. Fang, Y.-P. Wen, J.-Y. Zhou, Z.-J. Zhan, R. Ding, O. Li and H. Gao, Microb. Biotechnol., 2011, 4, 491-502.

41 C.-D. Qian, X.-C. Wu, Y. Teng, W.-P. Zhao, O. Li, S.-G. Fang, Z.-H. Huang and H.-C. Gao, Antimicrob. Agents Chemother., 2012, 56, 1458-1465.

42 G. Hertlein, S. Müller, E. Garcia-Gonzalez, L. Poppinga, R. D. Süssmuth and E. Genersch, PLoS One, 2014, 9, e108272.

43 J. Y. Lee, K. D. Passalacqua, P. C. Hanna and D. H. Sherman, PLoS One, 2011, 6, e20777.

44 B. Bister, D. Bischoff, G. J. Nicholson, M. Valdebenito, K. Schneider, G. Winkelmann, K. Hantke and R. D. Süssmuth, Biometals, 2004, 17, 471-481.

45 D. Segond, E. Abi Khalil, C. Buisson, N. Daou, M. Kallassy, D. Lereclus, P. Arosio, F. Bou-Abdallah and C. Nielsen Le Roux, PLoS Pathog., 2014, 10, e1003935.

46 S. Cendrowski, W. MacArthur and P. Hanna, Mol. Microbiol., 2004, 51, 407-417.

47 S. Sood, H. Steinmetz, H. Beims, K. I. Mohr, M. Stadler, M. Djukic, W. von der Ohe, M. Steinert, R. Daniel and R. Müller, Chembiochem, 2014, 15, 1947-1955.

48 R. Maget-Dana and F. Peypoux, Toxicology, 1994, 87, 151174.

49 K. Tsuge, T. Akiyama and M. Shoda, J. Bacteriol., 2001, 183, 6265-6273.

50 Z. Ma, N. Wang, J. Hu and S. Wang, J. Antibiot., 2012, 65, 317322.

51 R. H. Baltz, V. Miao and S. K. Wrigley, Nat. Prod. Rep., 2005, 22, 717-741.

52 L. Thimon, F. Peypoux, J. Wallach and G. Michel, FEMS Microbiol. Lett., 1995, 128, 101-106.

53 D. Romero, M. F. Traxler, D. López and R. Kolter, Chem. Rev., 2011, 111, 5492-5505.

54 R. I. Aminov, Environ. Microbiol., 2009, 11, 2970-2988.

55 E. Garcia-Gonzalez, S. Müller, P. Ensle, R. D. Süssmuth and E. Genersch, Environ. Microbiol., 2014, 16, 1297-1309.

56 M. A. Marahiel, T. Stachelhaus and H. D. Mootz, Chem. Rev., 1997, 97, 2651-2674.

57 M. A. Wyatt, W. Wang, C. M. Roux, F. C. Beasley, D. E. Heinrichs, P. M. Dunman and N. A. Magarvey, Science, 2010, 329, 294-296.

58 M. A. Wyatt, W. Wang, C. M. Roux, F. C. Beasley, D. E. Heinrichs, P. M. Dunman and N. A. Magarvey, Science, 2011, 333, 1381.

59 E. Garcia-Gonzalez, S. Müller, G. Hertlein, N. Heid, R. D. Süssmuth and E. Genersch, MicrobiologyOpen, 2014, 3, 642-656.

60 S. Müller, E. Garcia-Gonzalez, A. Mainz, G. Hertlein, N. C. Heid, E. Mösker, H. van den Elst, H. S. Overkleeft, E. Genersch and R. D. Süssmuth, Angew. Chem., Int. Ed., 2014, 53, 10821-10825.

61 Y. A. Chan, M. T. Boyne, A. M. Podevels, A. K. Klimowicz, J. Handelsman, N. L. Kelleher and M. G. Thomas, Proc. Natl. Acad. Sci. U. S. A., 2006, 103, 14349-14354.

62 Y. A. Chan and M. G. Thomas, Biochemistry, 2010, 49, 36673677. 
63 G. Weber, K. Schörgendorfer, E. Schneider-Scherzer and E. Leitner, Curr. Genet., 1994, 26, 120-125.

64 J. S. Scott-Craig, D. G. Panaccione, J. A. Pocard and J. D. Walton, J. Biol. Chem., 1992, 267, 26044-26049.

65 M. J. Kobylarz, J. C. Grigg, S. J. Takayama, D. K. Rai, D. E. Heinrichs and M. E. P. Murphy, Chem. Biol., 2014, 21, 379-388.

66 H. Beiderbeck, K. Taraz, H. Budzikiewicz and A. E. Walsby, Z. Naturforsch., C: J. Biosci., 2000, 55, 681-687.

67 N. M. Llewellyn, Y. Li and J. B. Spencer, Chem. Biol., 2007, 14, 379-386.

68 D. Reimer, K. M. Pos, M. Thines, P. Grün and H. B. Bode, Nat. Chem. Biol., 2011, 7, 888-890.

69 D. Reimer and H. B. Bode, Nat. Prod. Rep., 2014, 31, 154-159.

70 R. R. Forseth, S. Amaike, D. Schwenk, K. J. Affeldt, D. Hoffmeister, F. C. Schroeder and N. P. Keller, Angew. Chem., Int. Ed., 2013, 52, 1590-1594.

71 P. Belin, M. Moutiez, S. Lautru, J. Seguin, J.-L. Pernodet and M. Gondry, Nat. Prod. Rep., 2012, 29, 961-979.

72 K. Blin, M. H. Medema, D. Kazempour, M. A. Fischbach, R. Breitling, E. Takano and T. Weber, Nucleic Acids Res., 2013, 41, W204-W212.

73 C. Corvey, T. Stein, S. Düsterhus, M. Karas and K.-D. Entian, Biochem. Biophys. Res. Commun., 2003, 304, 48-54.

74 M. C. Booth, C. P. Bogie, H. G. Sahl, R. J. Siezen, K. L. Hatter and M. S. Gilmore, Mol. Microbiol., 1996, 21, 1175-1184.

75 A. Marchler-Bauer, S. Lu, J. B. Anderson, F. Chitsaz, M. K. Derbyshire, C. DeWeese-Scott, J. H. Fong, L. Y. Geer, R. C. Geer, N. R. Gonzales, M. Gwadz, D. I. Hurwitz, J. D. Jackson, Z. Ke, C. J. Lanczycki, F. Lu, G. H. Marchler, M. Mullokandov, M. V. Omelchenko, C. L. Robertson,
J. S. Song, N. Thanki, R. A. Yamashita, D. Zhang, N. Zhang, C. Zheng and S. H. Bryant, Nucleic Acids Res., 2011, 39, D225-D229.

76 M. Maqueda, M. Sánchez-Hidalgo, M. Fernández, M. Montalbán-López, E. Valdivia and M. Martínez-Bueno, FEMS Microbiol. Rev., 2008, 32, 2-22.

77 R. E. Wirawan, K. M. Swanson, T. Kleffmann, R. W. Jack and J. R. Tagg, Microbiology, 2007, 153, 1619-1630.

78 P. G. Arnison, M. J. Bibb, G. Bierbaum, A. A. Bowers, T. S. Bugni, G. Bulaj, J. A. Camarero, D. J. Campopiano, G. L. Challis, J. Clardy, P. D. Cotter, D. J. Craik, M. Dawson, E. Dittmann, S. Donadio, P. C. Dorrestein, K.-D. Entian, M. A. Fischbach, J. S. Garavelli, U. Göransson, C. W. Gruber, D. H. Haft, T. K. Hemscheidt, C. Hertweck, C. Hill, A. R. Horswill, M. Jaspars, W. L. Kelly, J. P. Klinman, O. P. Kuipers, A. J. Link, W. Liu, M. A. Marahiel, D. A. Mitchell, G. N. Moll, B. S. Moore, R. Müller, S. K. Nair, I. F. Nes, G. E. Norris, B. M. Olivera, H. Onaka, M. L. Patchett, J. Piel, M. J. T. Reaney, S. Rebuffat, R. P. Ross, H.-G. Sahl, E. W. Schmidt, M. E. Selsted, K. Severinov, B. Shen, K. Sivonen, L. Smith, T. Stein, R. D. Süssmuth, J. R. Tagg, G.-L. Tang, A. W. Truman, J. C. Vederas, C. T. Walsh, J. D. Walton, S. C. Wenzel, J. M. Willey and W. A. van der Donk, Nat. Prod. Rep., 2013, 30, 108-160.

79 H. Abriouel, C. M. A. P. Franz, N. Ben Omar and A. Gálvez, FEMS Microbiol. Rev., 2011, 35, 201-232.

80 X.-H. Chen, A. Koumoutsi, R. Scholz and R. Borriss, J. Mol. Microbiol. Biotechnol., 2009, 16, 14-24.

81 T. Carver, N. Thomson, A. Bleasby, M. Berriman and J. Parkhill, Bioinformatics, 2009, 25, 119-120. 